\title{
MATERIAL Y MEMORIA: TRANSFORMACIONES EN LA OBRA DE LUIS BARRAGÁN
}

\author{
Carlos Labarta Aizpún
}

No son pocos los arquitectos que, tras abrazar la modernidad, encontraron su particular conversión desde la experiencia táctil y espiritual del material o, incluso, desde la evocación de su memoria y nostalgia. A todos ellos les llegó un momento en el que los tensos planos enfoscados en blanco les parecían de una excesiva abstracción y, sobre todo, distantes de otras realidades materiales por las que sentían una atracción más honda y permanente, y que activaban, simultáneamente, su memoria y las razones espirituales del construir. De hecho, en esa blanca abstracción no se mantuvieron ni sus creadores. Uno de los conversos de más largo recorrido es Barragán y las transformaciones en su obra son deudoras de los múltiples encuentros con los materiales y sus paisajes.

Palabras clave: Revisión, modernidad, experiencia material, memoria, soledad Keywords: Revision, modernity, material experience, memory, solitude
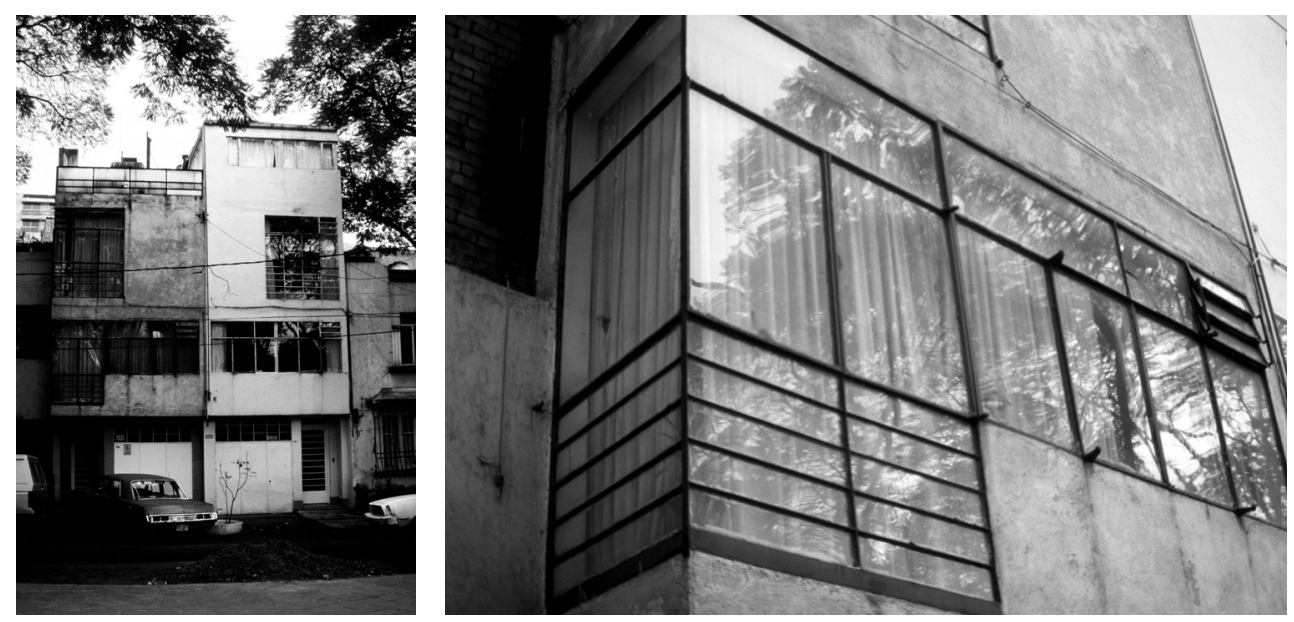

Fig. 1. BARRAGÁN, Luis. Casas en la Avenida Parque Méjico, Méjico D.F., 1936. (Fotografía C. Labarta).

Fig. 2. BARRAGÁN, Luis. Detalle de la fachada. Casas en la Avenida Parque Méjico, Méjico D.F., 1936. (Fotografía C. Labarta).

MATERIALES IMPORTADOS: EL MOVIMIENTO MODERNO COMO HISTORIA RECIENTE Y CATÁLOGO

Tras los primeros años de trabajos profesionales en su Guadalajara natal, en los que el arquitecto refiere a búsquedas locales y a la aplicación de su primer aprendizaje, Barragán realiza su primer viaje a Europa en 1924 y queda impresionado por la modernidad recién formulada. Desde ese momento se vuelca en el análisis y aplicación de las nuevas verdades arquitectónicas. Barragán en 1935 ve cumplido su sueño de instalarse en M éjico D.F. Su llegada a la capital coincide con el auge de la modernización cultural de país sometido a una creciente influencia de las tendencias modernas de Europa y N orteamérica. En este ambiente comienza a obtener los primeros encargos residenciales en un periodo que duraría hasta 1940 y en el que sus influencias arquitectónicas pueden encontrarse, principalmente, en el L e Corbusier de Ville Savoie, en las sencillas volumetrías de Oud y, genéricamente, en la manipulación de geometrías cúbicas y, sobre todo, blancas.

De este modo, hasta la fecha mencionada, Barragán participó en proyectos de viviendas aceptando la dimensión especulativa o comercial de la construcción. La influencia del movimiento moderno y la respuesta productiva a las demandas del momento forjaron en el arquitecto un panorama que puede ser comprendido tanto desde las afirmaciones por las que 


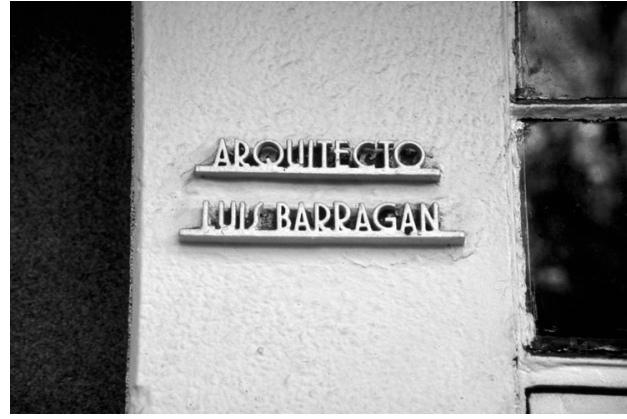
nida Parque Méjico, Méjico D.F., 1936. (Fotografía C. Labarta).
Fig. 3. BARRAGÁN, Luis. Detalle de la firma. Casas en la Ave-

apostaba como por las negaciones que éstas implicaban. La arquitectura hasta entonces se afirmaba en B arragán como: construcción; idea que puede ser construida en cual quier lugar; tecnología frente a arte. Se presentaba, a su vez, como una serie de negaciones: la construcción tradicional; el lugar, el paisaje; el sentido de habitar en un sentido existencial más complejo; arte, o mejor en vocabulario del propio arquitecto, serenidad, contemplación, soledad.

L a arquitectura moderna era reconocida por Barragán como un catálogo de postulados precisos y vocabulario reproducible. Como ejemplo de esta fase de su obra en la ciudad de M éjico (recordemos que anteriormente había trabajado en su Guadalajara natal en continuidad con la arquitectura col onial) cabe referir, entre otras, a las dos casas en la Avenida Parque M éjico, 1936 en las que, inconfundiblemente, se repite la retórica moderna ${ }^{1}$ (Figs. 1 y 2). Esa voluntad de transformación del mundo, enfática y final mente fallida, que al entó a los arquitectos modernos, se expresa en esa firma que, grabada en hierro, se exhibe en la fachada (Fig. 3). N unca más volvería B arragán a firmar sus obras de este modo. Solamente la utilización de las referencias externas sería objeto de este pequeño gesto de vanidad; las verdades por descubrir permanecerán en el anonimato. Hasta el material del acero sería abandonado. Como si los materiales importados no tuvieran la capacidad de transmitir, de conducir, de reflejar las realidades a las que la arquitectura tenía que dar, no solo respuesta, sino cobijo.

También Le Corbusier, tempranamente confrontado con la realidad de sus viajes a L atinoamérica y a España a finales de la década de los veinte e inicios de los treinta, dudó de la estricta permanencia en los materiales característicos del inicio de la modernidad -el vidrio y el acero- para asumir, con la intuición propia de la inteligencia emocional, el resultado de esas experiencias sensibles del viaje. Por ello, cabe recordar que la contradicción, en relación con la materialidad, que algunos arquitectos modernos sintieron, tuvo su origen más explícito en el tiempo germinal de la propia modernidad. B asta recordar la evolución del propio L e Corbusier para comprender cómo, simultáneamente, los mencionados material es con los que se construía el proyecto moderno comenzaron a confundirse con aquellos extraídos del entorno. EI resultado, como es sabido, cristaliza en la aceptación y utilización de los materiales próximos, como la piedra, en la Casa M androt, Le Pradet (Toulon), 1929-1931. Y con la experiencia de la materia, no solo recupera para la arquitectura todo el mundo a su alrededor, sino que la convierte en factor de evolución de la propia modernidad. Porque en esta casa : "ya no hay solo un nuevo orden a través de un juego plástico sino que la materia empieza a adquirir una importancia que ya no abandonará en toda su vida"2.

El joven Barragán, con treinta y ocho años, en la cúspide de una fulgurante carrera profesional en la capital de su país, experimentó igualmente el desamparo de la materia inerte y repetida, desoladoramente distante 3 . A caso precisa, eficaz y productiva, pero incapaz de dar respuesta a los valores espirituales que, en el fondo, anidaban en la memoria del arquitecto llegado unos años antes de su provincia natal. En sus recuerdos se acumulaban los paisajes vividos y, con ellos, el olor, la textura y las ensoñaciones derivadas de los materiales que los conformaban.

Larza, 1939. Cuatro estudios de pint en la plaza Melchor Ocampo, 1939; Edificios de apartamentos en la calle Río Elba 38, 50, 52 y 56, 1936-1940; Edificio de apartamentos en la plaza Melchor Ocampo, 1936-1940.

2. ALONSO DEL VAL, Miguel Ángel. "A vueltas con la materia y la técnica. Una conversación con Miguel Ángel Alonso del Val", Revista ZARCH, n. 4, Departamento de Arquitectura Universidad de Zaragoza, 2015, p. 163.

3. Lo mismo le ocurrió a Dimitris Pikionis quien pasó, de las blancas y ortodoxas escuelas en el Licabeto, Atenas, 1933, a entretenerse durante años, jugando con las piedras, en los accesos a la acrópolis ateniense.

4. "A principios de los años 40 , harto una vez más de sobrellevar el trato difícil y soso que una clientela particular exigía al arquitecto de la época, Luis Barragán anuncia su retiro de la práctica pública de la profesión. Con algunas excepciones, de esta fecha en adelante, solo aceptaría trabajos en los que el cliente fuera él mismo. Lucidez, ¿legítima defensa? Lo cierto es que siente como la horda de los filisteos lo cerca. En una carta a Rafael Urzúa, muchos años antes, ya lo había dicho". Eguiarte, Gillermo. Luis Barragán, Reverte Ediciones, México, 1996, p. 112. Incluye textos de José María Buendía Júlbez, Juan Palomar, Guillermo Eguiarte, fotografías de Sebastián Saldivar y prólogo de Álvaro Siza.

\section{DEL ABANDONO DE LA ARQUITECTURA A SU REDEFINICIÓN: EL JARDÍN COMO MATERIAL Y OBJETO DEL PROYECTO}

Por ello Barragán, hacia 1940, decide abandonar la práctica de la arquitectura iniciando un profundo proceso que desembocará en su redefinición y expansión 4 . A bandonándola la redefine más allá de los límites estilísticos, funcionales y constructivos a los que, hasta entonces, se había referido. Y esta redefinición tiene su origen en el encuentro con el jardín como material y objeto de proyecto. Comienza en ese periodo a interesarse por su diseño y por la incorporación de sus lecciones a la arquitectura. En este encuentro se produce el punto de inflexión en su trayectoria. Su pasión por los jardines es fruto de sus recuerdos de las haciendas de su provincia natal así como del encuentro y descubrimiento del escritor y paisajista francés Ferdinand Bac a quien había conocido, un 16 de agosto de 1931, en su viaje a E uropa. En sus preferencias se diluyen, definitivamente, las verdades dogmáticas y crece el interés por el paisaje, la nostal gia, la memoria.

B arragán comienza a construir jardines en la antigua Tacubaya, zona de recreo de los habitantes de la ciudad de M éjico, actual mente absorbida por la trama de la extensa ciudad. En 


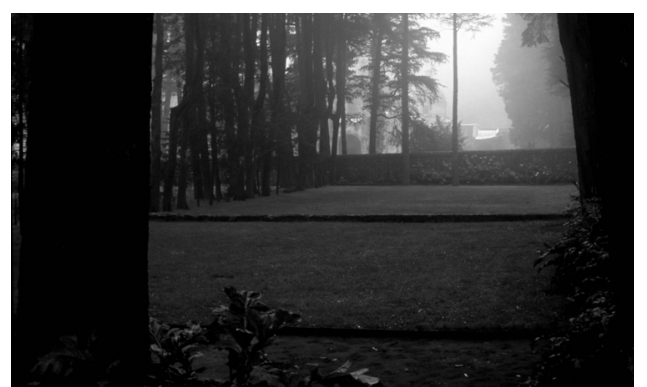

4

el mismo lugar donde se inició con los jardines construyó, unos años más tarde, dos sucesivas casas para sí mismo y su estudio. El diseño de los jardines supone la traducción paciente de los recuerdos de su infancia en las sierras de J alisco, de sus viajes por A ndalucía, de L es Colombières, de todos los espacios que su memoria incansablemente reproducía como evocación de la tradición anónima recibida y que él, con sus mismos materiales, continuaba.

A demás de los jardines de Tacubaya, Barragán descubrió el encanto de unos terrenos volcánicos, al sur de la ciudad, en la zona llamada El Pedregal. A quí halló otro espacio para su experimentación. La construcción de los jardines de EI Pedregal, 1945-1950, se solapó en el tiempo con la construcción de la primera casa en ellos, la Casa Prieto. En los jardines B arragán consigue la belleza a través de la unidad entre el paisaje y la expresión estética. L o relata en su discurso de aceptación del Premio Pritzker:

"Cuando me encontraba en un campo de lava al sur de la ciudad de M éjico, asombrado por la belleza de ese paisaje volcánico, me propuse realizar al gunos jardines que hicieran ese lugar habitable y maravilloso y lo complementaran. Caminaba entre grietas de lava, altos muros de roca, para salir, con gran sorpresa, a hermosos valles de las más hermosas y caprichosas formas fantásticas" 5 .

El arquitecto funde, de este modo, su obra con la tierra de la que surge, construyendo con los materiales del lugar y domesticando la naturaleza. Su intención es completarla, sin violentarla, según la tradición heredada también de los monasterios mejicanos (Fig. 4). En ellos descubre el valor de la huella como impronta del hombre en el medio natural. El suave fraccionamiento de los jardines mediante una quebrada línea dibujada entre ellos será reinterpretada por el arquitecto en EI Pedregal (Fig. 5). N uevamente la evocación de la memoria y de una recuperada materialidad late en el proyecto. Su objetivo, en este su primer jardín, no es otro que complementar y nivelar diversas plataformas para crear un jardín en compartimentos, recordando la belleza de los jardines y patios de la A lhambra y del Generalife que visitara en su viaje a España.

Probablemente cuando B arragán escribe, en el año 1931, "hay que buscar que las casas sean jardines, y los jardines sean casas", quiere expresar su deseo de incorporar en sus moradas la doctrina de Bac que yace detrás de sus jardines ${ }^{6}$. Por eso no es de extrañar que, cuando tuvo ocasión de convertir en realidad estos anhelos, el arquitecto se entregase devocionalmente a ello. De mi visita a la Casa Prieto, en el ya lejano año de 1991, guardo nítidos recuerdos. Y, a los efectos que nos ocupan, rememoro cómo su propietario, D. Eduardo Prieto, narraba la decisiva influencia y atracción que la materia ejercía sobre el arquitecto quien, en sus viajes, portaba tierra y plantas de otros lugares próximos para el diseño del jardín (Fig. 6).

Tras el grueso muro de piedra, única referencia de la vivienda con la calle, se accede a un patio empedrado (Fig. 7). Tras su etapa de filiación a la arquitectura moderna Barragán inicia con esta casa, asociada a los materiales tradicionales, una fase de interiorización que ya no abandonará hasta su muerte, acaecida cuarenta y tres años después. L a recurrencia a la piedra, la madera y los enfoscados tradicionales no solo surge por la necesidad de evocar su propia historia sino por la voluntad de convocar en estos materiales la nostal gia de otras culturas.

L a atmósfera creada en esta vivienda precisa del concurso amable y natural de la materia, y de su experimentación, para comprenderse en toda su intensidad. Y alcanza su condición

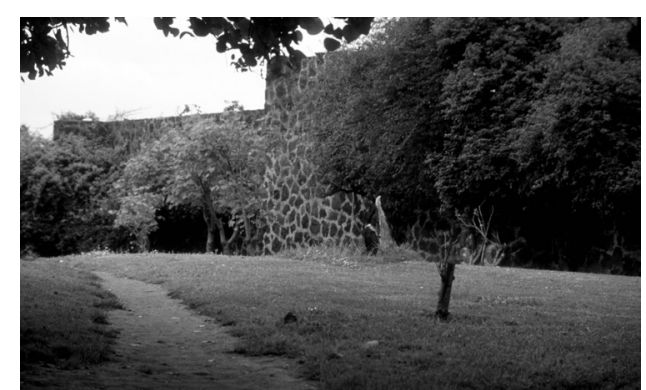

6

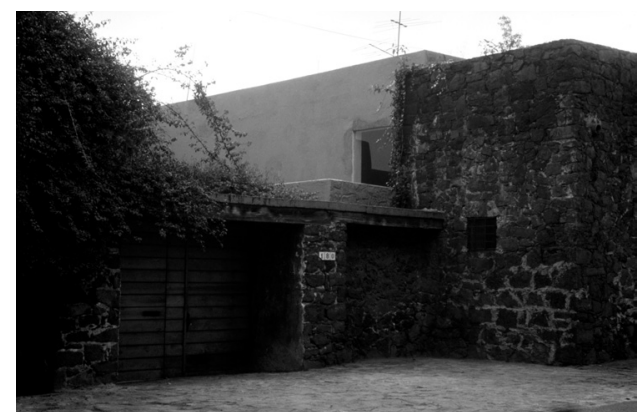
7

Fig. 4. Monasterio en Méjico D.F., siglo XVI, vista de los jardines. (Fotografía C. Labarta).

Fig. 5. BARRAGÁN, Luis. Jardines de El Pedregal, Méjico D.F., 1945-1950. (Fotografía C. Labarta).

Fig. 6. BARRAGÁN, Luis. Jardín interior, Casa Prieto, El Pedregal, Méjico D.F., 1945-1950. (Fotografía C. Labarta).

Fig. 7. BARRAGÁN, Luis. Fachada de acceso, Casa Prieto, El Pedregal, Méjico D.F., 1945-1950. (Fotografía C. Labarta).

5. BARRAGÁN, Luis. Discurso de aceptación. Premio Pritzker, Dumbarton Oaks, Washington D.C., 3 de junio de 1980.

6. Cfr., CURIEL GÁMEZ, Fernando. La biblioteca de Luis Barragán: 1925-1980. Tesis doctoral inédita. Escuela de Arquitectura de la Universidad de Navarra, 2012. 

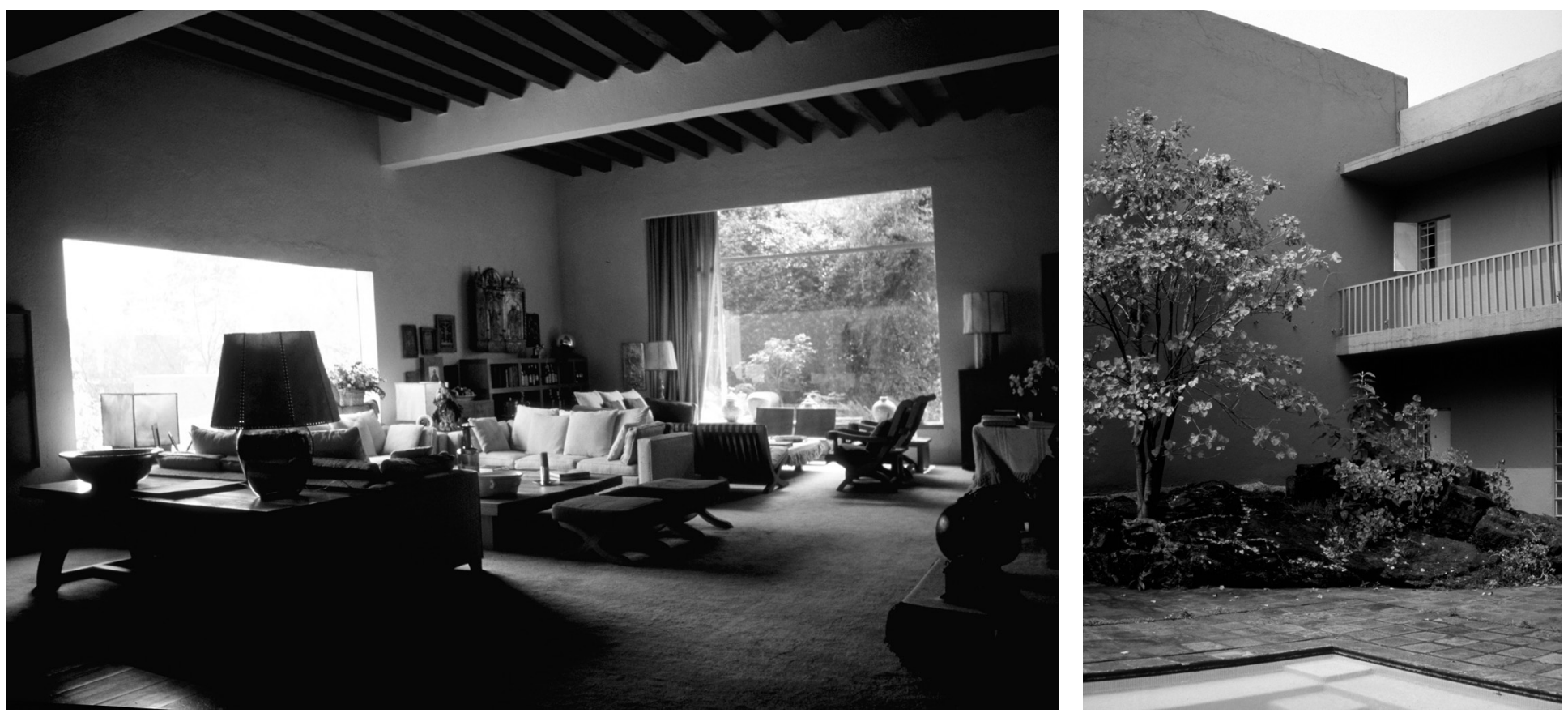

8

Fig. 8. BARRAGÁn, Luis. Vista de la sala de estar, Casa Prieto, El Pedregal, Méjico D.F., 1945-1950. (Fotografía C. Labarta).

Fig. 9. BARRAGÁN, Luis. Fachada al jardín interior, Casa Prieto, El Pedregal, Méjico D.F., 1945-1950. (Fotografía C. Labarta). de permanencia precisamente en el sabio uso de los materiales. La habitación del hombre moderno ya no era una máquina para vivir sino una casa donde encontrarse. A sí, por ejemplo, el espacio principal, como en las casas de campo, lo preside la chimenea invadiéndolo con el aroma de la leña de mezquite arrancada de los campos mejicanos (Fig. 8).

L os materiales, indistintamente, se utilizaban en la casa y en su jardín. Todo el terreno donde se asentaba la vivienda no era productivo y, paulatinamente se iba descrestando la lava, para acomodar la vivienda a las condiciones de aquél (Fig. 9). Del diseño de los jardines próximos de EI Pedregal Barragán extrajo descubrimientos como la planta denominada pirus, capaz de desarrollarse entre las lavas y que fue utilizada para el diseño del jardín. Las combinaciones de vegetación eran múltiples pero Barragán prefería una particularmente: níspero, clavo y piracanto. El arquitecto evitó un diseño medido y encorsetado del jardín, permitiendo que la información suministrada por la propia vegetación se fundiese con la amable naturalidad de los espacios interiores.

En una arquitectura basada en la percepción sensorial y en la experiencia táctil de la materia cada detalle debía ser minuciosamente examinado por el arquitecto. El olor, el tacto, el color juegan un papel fundamental en el conocimiento de la vivienda. A tal extremo llegaba la autenticidad del proceso y del modo de aplicación de los materiales empleados, que, por ejemplo, la pintura debía de ser de cal con colores naturales. El arquitecto odiaba literal, y visceralmente, otras aplicaciones. Como si la sinceridad del material fuera la suya propia. Siguiendo la tradición hispana de épocas anteriores B arragán añadía a la cal unas pencas de magueil - planta de la región- y sal, con el fin de espesar la mezcla y obtener unos paramentos densos al tacto y a la vista. Densidad del material que define el carácter de la obra de Barragán como nos recuerda Siza: "U na arquitectura que nos envuelve como presencia física, simple y densa, imposible de describir o de imitar o de fotografiar; universal y actual" 7 . Con el tiempo se comercializaron el acrílico y el vinílico que ofrecían unas texturas similares a la cal. El propietario, quizá en su única "traición" a su entrañable amigo Barragán, pintó unas superficies con la pintura sintética. Tras varios meses sin que el arquitecto percibiese nada extraño el propietario le comentó su osadía. Con la humildad de los maestros Barragán aceptó el reto de su amigo utilizando desde entonces las pinturas.

\section{ASPIRACIONES ESPIRITUALES CON EL CONCURSO DE LA MATERIA}

Barragán, en definitiva, aspira, como Ferdinand Bac, a expresar con la materia el senti-

miento de vinculación y refugio del hombre con y en la naturaleza. En referencia a su crea-
7. SIZA, Álvaro. Álvaro Siza Textos, edición de Carlos Campos Morais, Abada Editores, Madrid, 2014, p. 156 
ción de Les Colombiéres, B ac revela que su ambición no es otra que la de expresar con la materia un sentimiento común a muchos hombres que buscan un vínculo con la naturaleza, creando un lugar de reposo, de placer apacible. Uniéndose, de ese modo, a la solidaridad milenaria a la que todos estamos sujetos. Y para tan noble cometido el arquitecto no cuenta sino con la pobreza, pero también con la autenticidad, de la materia desnuda aprehendida entre campos de lava y jardines habitados.

Porque, en el fondo, Barragán encuentra en la tierra su materia espiritual. Y querría, si ello fuera posible, que de ella brotasen, sin transformación, todos los elementos constructivos. De manera que, la memoria de los lugares igualmente por la materia perpetuada, configurase el cobijo del nuevo hombre moderno que solo puede serlo en la medida en que asume, antropológicamente, su evolución desde la tradición. Como nos relata Felipe L eal: "a pesar de haber estudiado ingeniería, sus mayores inquietudes no estuvieron al lado de las técnicas construc-
tivas sino de las experiencias formales y simbólicas enriquecedoras del espíritu. Su arquitectura no la concebía
como una arquitectura para la arquitectura, sino como una arquitectura realizada para la contemplación" ${ }^{8}$.

Barragán diluye, con su obra, la frontera entre las inquietudes técnicas y las humanísticas pal pando, en cada paramento, en cada pavimento, en cada objeto, las razones espiritual es del construir. En esta disolución también la materia sirve a la continuidad. ¿Cómo comprender, sino, que el muro en el patio del Convento de las Capuchinas de Tlalpan, M éjico 1952-1955, sea, simultáneamente, cerramiento y cruz? El muro se pliega para hacerse cruz (Fig. 10). La cruz nace del muro y permanece en él, materializando el permanente esfuerzo del arquitecto por conciliar todos los elementos constructivos en un discurso continuo y unitario, de tal forma que ningún elemento pueda ser percibido individualmente. El muro puede ser estructura, pero también cerramiento. Puede ser construcción, pero, también, y fundamentalmente, arte y paisaje. 0 , ¿cómo comprender, igual mente, que los cántaros, en el patio que conecta el estudio con el jardín de su casa en Tacubaya, 1948, no tengan base? A I ser huecos, cuando se cubre de agua toda la superficie del patio, se reflejan y, a la vez, reflejan, sin discontinuidad alguna, el cielo. Experiencia que se extiende al patio de la casa Gilardi, 1976 (Fig. 11). 0 ¿por qué los jarrones de la casa están a medio llenar de tal manera que reflejan, invertido, el paisaje exterior? Por la misma razón por la que las esferas se disponen, con sus superficies pulidas, por todas las estancias para reverberar las superficies y destellos del todo el espacio al rededor. En un intento úl timo de que la materia contribuya, exclusivamente, a la reconciliación con el paraíso perdido, con el jardín eterno que, el arquitecto, con sus construcciones, querría anticipar.

Esta continuidad del material se torna en continuidad y concatenación espacial de tal modo que su arquitectura permite la vivencia de una secuencia encadenada, entre instantes ilimitados y, por tanto, imperceptibles individualmente. Permite un curso fluvial, cambiante, pero ininterrumpido. Bien lo recuerda Siza en referencia a la obra de B arragán: "A I guien nos conduce por los espacios. N os deslizamos" ${ }^{\prime \prime}$. Es esta condición de deslizamiento y continuidad la que permite el disfrute gozoso, sin sobresaltos, de una obra que precisa para ello, de materiales igualmente moldeables.

A simismo, envueltos en una presencia física determinada por los materiales desnudos, su obra ofrece comprensión y cobijo. La arquitectura contemporánea no nos acoge. Es reflejo del mundo inhóspito que nos rodea. Quetglás ha escrito sobre esta condición inhospitalaria:

\footnotetext{
"L as cosas que hay a nuestro alrededor no nos acogen, no permiten que vayamos hasta ellas para apoyarnos. Son riquísimas, hermosas, vivas - pero no nos aceptan-. Cézanne decía de las cosas que eran "esféricas". Veía esferas, conos y cilindros por todas partes, en cualquier sitio donde enviara su mirada. Un mundo hecho todo él de superficies convexas, abarquilladas, de objetos vueltos de espalda, donde no hay abierta ninguna concavidad para recoger nuestra mirada. Un mundo sin apoyos, resbaladizo, de escamas; una disgregación desasistida, sin frente, por donde se despeña nuestra mirada, hasta venir al suelo. A ese caer a tierra, a ese mundo que nunca nos recibe, le llamamos moderno" ${ }^{10}$.
}

Por el contrario, la arquitectura de Barragán, de superficies planas, es cóncava y convexa a un tiempo. Porque no nos rechaza, sino que recoge nuestra mirada, y tras ella a nosotros mismos y a nuestros objetos, diluyendo en ese momento cualquier límite físico (Fig. 12). L a arquitectura de nuestro tiempo ha querido, en vano, diluir sus límites físicos y ha doblado indefinidamente sus superficies, en un ejercicio pendular entre lo cóncavo y lo convexo, en

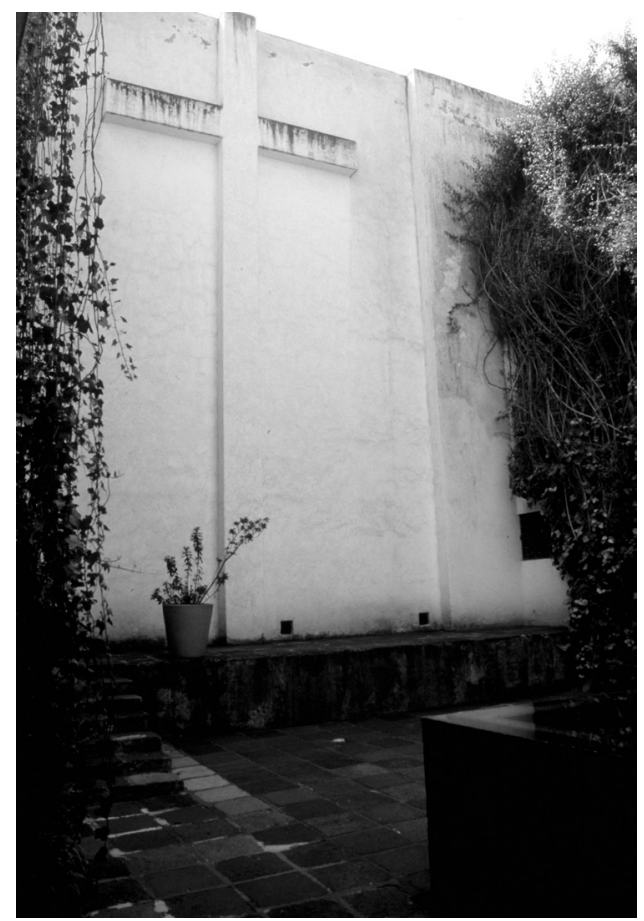

10

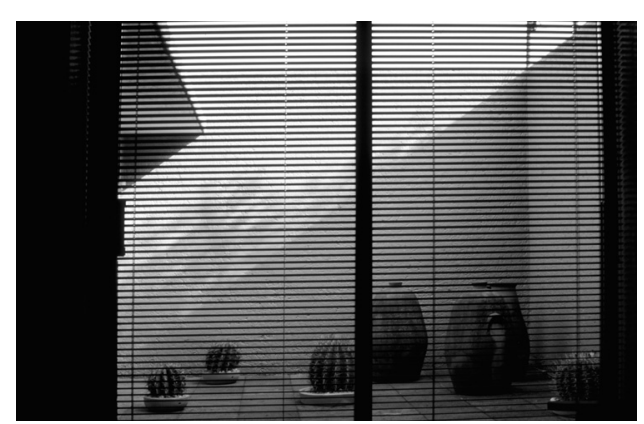

11

Fig. 10. BARRAGÁN, Luis. Vista del patio, Convento de las Capuchinas, Tlalpan, Méjico, 1952-1955. (Fotografía C. Labarta). Fig. 11. BARRAGÁN, Luis. Vista del patio, Casa Gilardi, Méjico D.F., 1976. (Fotografía C. Labarta)

8. LEAL, Felipe. “Una tarde en la casa de Barragán”, recogido en AA.VV., En el mundo de Luis Barragán, Artes de México, México, 1999, p. 67.

9. SIZA, Álvaro. Álvaro Siza Textos, p. 156.

10. QUETGLÁS, Josep. "Pasado a limpio II", Pre-textos de Arquitectura, edición al cuidado de Carles Muro, Inés de Rivera y Ton Salvadó, Demarcació de Girona, Col-legi d'Arquitectes de Catalunya, p. 164 


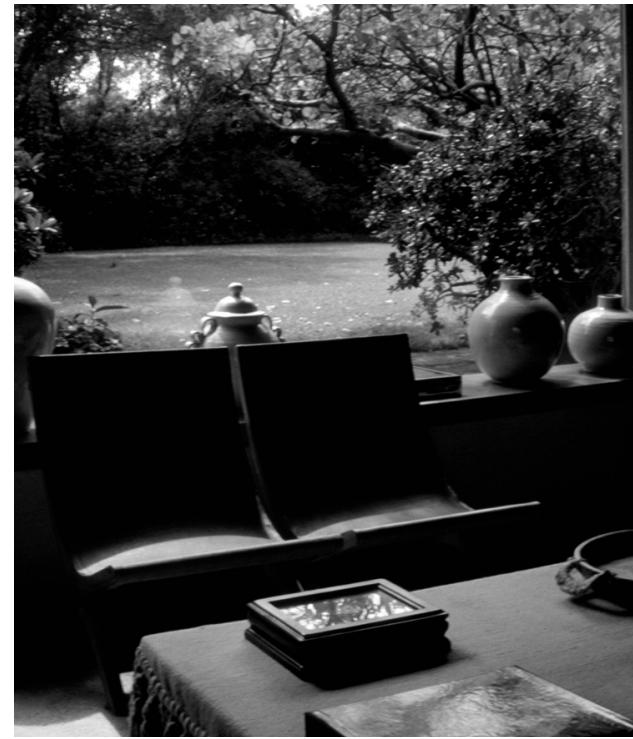

12

Fig. 12. BARRAGÁn, Luis. Detalle de la sala de estar, Casa Prieto, El Pedregal, Méjico D.F., 1945-1950. (Fotografía C. Labarta). Fig. 13. Monasterio en Méjico D.F., siglo XVI, detalle de entrada de luz. (Fotografía C. Labarta).

Fig. 14. BARRAGÁN, Luis. Detalle de entrada de luz en e baño, Casa Gilardi, Méjico D.F., 1976. (Fotografía C. Labarta).

11. "Al señor Barragán, a quien me gustaría llamarle mi nieto, por la perfecta comprensión que él tiene de mi Renovación de un estilo mediterráneo y español". Traducción del autor.

12. CURIEL GÁMEZ, Fernando. La biblioteca de Luis Barragán, p. 12.

13. Recogido por Kenneth Frampton en Historia crítica de la arquitectura moderna, Gustavo Gili, Barcelona, 1987, p. 323.
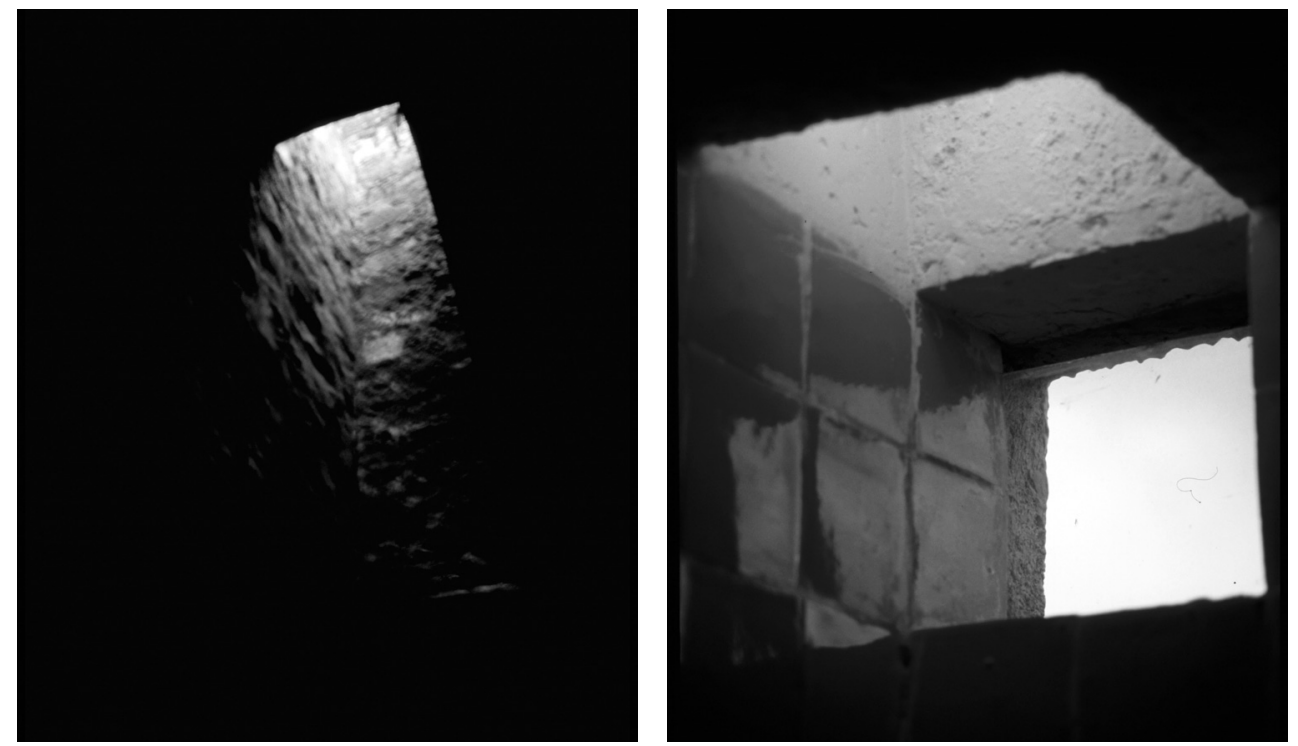

13

14

un intento de liberación de los dos momentos extremos, sin conseguirlo. Barragán, en una lección oculta, no se sitúa en ninguno de los dos extremos sino que, liberándose de ambos, derriba los límites físicos sin necesidad de descomponerlos.

\section{EL MATERIAL COMO PORTADOR DE LA MEMORIA}

Se consolida en el arquitecto, de este modo, un rechazo de la tentación de la novedad, así como del estilo, para adentrarse en la arquitectura, de forma desnuda, sin más materia que la próxima - la piedra, la tierra, la madera, el agua- , con la única contribución de un elemento de nostal gia sostenida en el tiempo, precisamente, por la evocación de los materiales (Fig. 13). Nostal gia que, a su vez, reverbera los ecos derivados de la experiencia. En ellos, en la recreación de su nostal gia, basa el arquitecto su obra. De hecho, para B arragán, la arquitectura no es sino la materialización de esa nostalgia (Fig. 14).

Esta revolución silenciosa se nutre de las lecturas del escritor francés. Dos son los libros que Barragán ya nunca abandonaría: J ardins enchantés y Les Colombières. A tal extremo llega la identificación de Barragán con estas enseñanzas que, según leemos en la mencionada tesis doctoral de Fernando Curiel Gámez, guardará escrupulosamente los ejemplares en su biblioteca personal hasta el final de su vida. En ellos se lee la dedicatoria: "A M onsieur Barragan que je voudrais appeler mon filleul par la parfaite compréhension qu'il a de ma Renovation d'un style mediterranéen et espagnol" (Ferdinand Bac, 16 A oût, 1931)"11.

La obra de L es Colombiéres representa para Bac una búsqueda tempestuosa de aquellas nostál gicas regiones inverosímiles material izadas en ella, pero sin llegar a la perfección. No es la perfección el objetivo a conseguir. Como nos recuerda Fernando Curiel:

“Para el autor, la gran frustración humana reside en reproducir aquellas nostal gias que llevamos dentro de
nuestro ser, en su más fiel definición. Sin embargo, pese a la dramática imposibilidad de que el ser humano
aspire a verificar y materializar estas regiones inverosímiles, yace en él la esperanza de imitar estas formas e
instaurarlas en su estilo de vida, en una fertilidad inspirada por la sencillez, la paz interior y la naturaleza"12.

Al descubrimiento de esas nostalgias de todos los lugares vividos dedicó Barragán su vida, conocedor, como arquitecto constructor en el sentido más noble del término, que precisaba del insustituible concurso de la materia para poder revelarlas.

De esta condición del valor de la memoria, asociada a la vivencia del material, nos habla el propio Barragán. Su conocido texto autobiográfico resume su vinculación espiritual con los el ementos constructivos y con su materialidad, que concluye con la célebre sentencia: "No, no hay fotos, sólo tengo su recuerdo" ${ }^{13}$. A ntes nos ha paseado entre los material es de su infancia que, impregnados en su espíritu, le pertenecen y acompañan: 


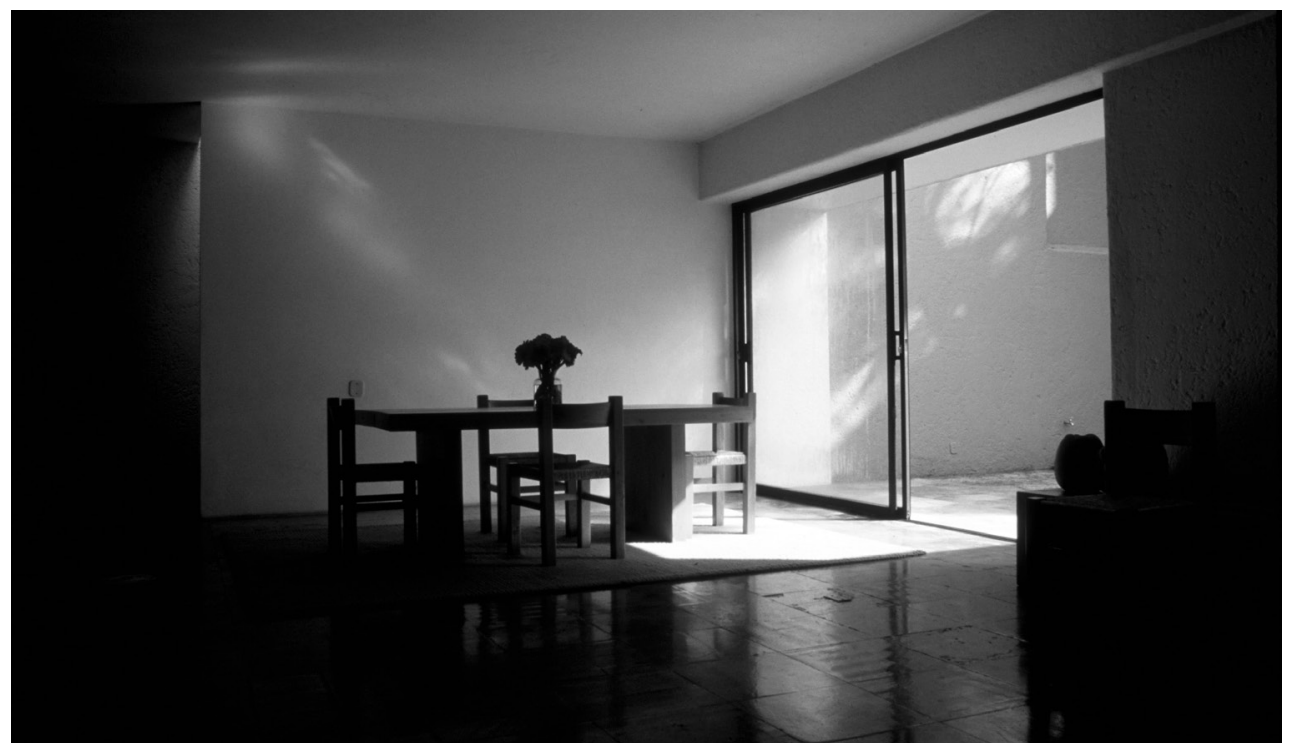

15

"M is recuerdos de la primera infancia se remiten al rancho que mi familia poseía cerca del pueblo de $\mathrm{M}$ azamitla. Era un pueblo de colinas, formado por casas con cubiertas de tejas y grandes aleros que protegían a los transeúntes de las pesadas Iluvias que caen en aquella región. Hasta el color de la tierra era interesante porque era tierra roja. En este pueblo, el sistema de distribución del agua consistía en grandes canalones formados por troncos, los cuales se apoyaban en una estructura de forcas de madera, de cinco metros de al tura por encima de los tejados. Este acueducto cruzaba sobre todo el pueblo regando los patios, en los que había grandes fuentes de piedra para recibir el agua. L os patios de las casas alojaban los establos en los que se mezclaban las vacas y las gallinas. Fuera, en la calle, donde había anillas de hierro para atar los caballos, el canalón cubierto de musgo, chorreaba agua sobre el pueblo. Tenía un ambiente de cuento de hadas"14.

El recuerdo, empapado de materialidad, nutre la memoria y, desde ella, proyecta el arquitecto. Entre estos recuerdos surge la interpretación de la hacienda mejicana y el arquitecto nos dispone, en sus diferentes obras, a la introversión desde el espacio de la calle y a la fluida transición hacia los espacios de la casa. B arragán suma a su obra la huella de los lugares visitados, y admirados. Esta manera de impregnar la arquitectura de la experiencia sensible del camino es patente en su etapa de madurez. La materia heredada contribuye, de este modo, a la creación de una arquitectura atemporal y universal. Porque en él la materia no se entiende como aquello opuesto al espíritu sino, paradójicamente, como el vehículo para su ensanchamiento.

En la arquitectura de B arragán, la materia, además de portar la memoria de los lugares, próximos y lejanos, contribuye a la condición de permanencia entendida como contribución a la construcción del orden espiritual del morador de cada una de las viviendas o espacios. Por ello la materialidad de cada una de las moradas permitiría que su ruina se fundiese de nuevo íntegramente en la tierra. U na arquitectura por tanto que, aunque se degradase, volvería a edificar la memoria de la que surgió.

El material igualmente se comprende en su obra como portador de las sensaciones de lo ordinario. Cuando el arquitecto utiliza los materiales próximos emanados de la tierra comprende la experiencia del hombre con y sobre ellos. Experiencia que, domésticamente, se ha ido forjando de manera laboriosa e inocente. B arragán con su obra pretende envolver y emular todas esas vivencias acumuladas. No precisa para ello de materiales excepcionales, en el sentido de deslumbrantes, distintos o ajenos, sino que, precisamente, encuentra, en la cotidianeidad de lo que le rodea, el material propicio para una arquitectura educada que podríamos llamar arquitectura de la naturalidad. A parentemente poco esforzada pero que goza de la sencillez propia de la sabiduría. Porque sabe que solamente de la experiencia de lo ordinario puede brotar la vivencia de lo extraordinario (Fig. 15).

Siza, a los efectos que estamos tratando en relación con la obra de B arragán, resume:

“N inguna innovación abandona la razón inmemorial. No hay innovación. Hay un reencuentro de la inocencia, una conquista del Estado de Gracia, para que no se pierda la M emoria"15.
Fig. 15. BARRAGÁN, Luis. Vista de la alberca, Casa Gilardi, Méjico D.F., 1976. (Fotografía C. Labarta)
14. Op.cit.

15. SIZA, Álvaro. Álvaro Siza Textos, p. 157.

16. PLAZAOLA, Juan, recogido por Araujo, Ignacio, Luz y proyecto, Apuntes del curso de Proyectos II, curso 1999-2000, Escuela Técnica Superior de Arquitectura, Universidad de Navarra, p. 24. 
Fig. 16. BARRAGÁN, Luis. Vista de la celosía del patio, Convento de las Capuchinas, Tlalpan, Méjico, 1952-1955. (Fotografía C. Labarta).

Fig. 17 BARRAGÁn, Luis. Detalle de la celosía del patio, Convento de las Capuchinas, Tlalpan, Méjico, 1952-1955. (Fotografía C. Labarta).

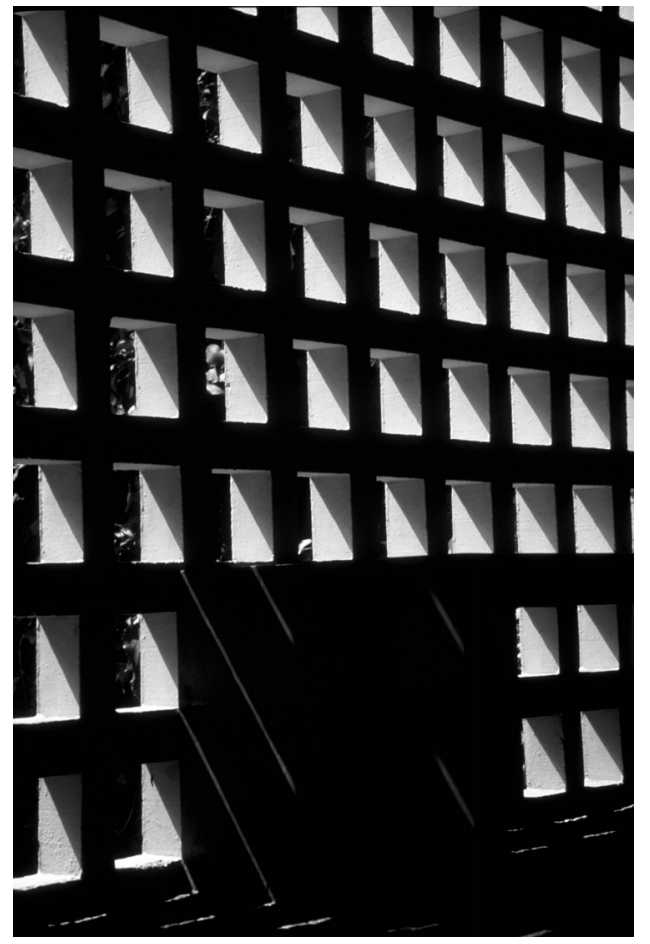

16

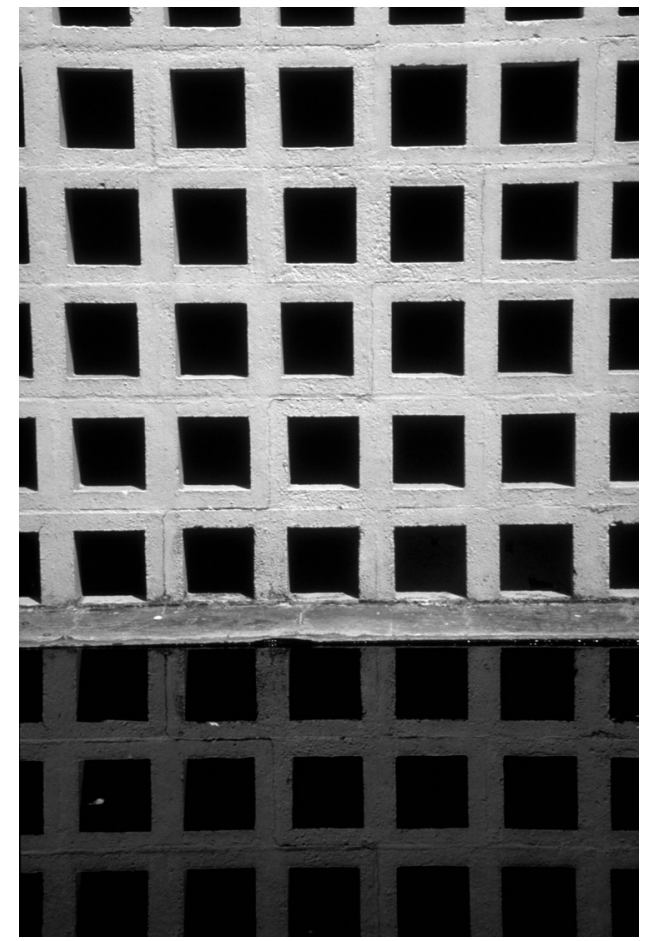

17

\section{MATERIAL Y TIEMPO, DUDA Y LUZ}

Los materiales tienen la capacidad de contribuir a una medida del tiempo porque permiten leer, sobre ellos, el paso de la luz. Y con ella, el del propio tiempo. Hay materiales que permiten la duda sobre ellos. Otros no. B arragán, como los artesanos, solamente construyó con los primeros. Y de ese modo adivinó la estructura precisa para la celosía del patio del mencionado Convento de las Capuchinas en la que se esconde una lección de la acción de la luz sobre la materia. B arragán encuentra huellas para su arquitectura en los antiguos conventos mejicanos. La relación de la luz con la profundidad de los claustros es una de ellas. Esta lección se condensa en el tapiz tramado o celosía que separa el patio del paso a la sacristía. Puede parecernos que esta imagen es conocida. No deberíamos pasar sobre ella con tanta celeridad. En ella la luz define, a cada instante, el espacio. L as sombras se dibujan en el suelo, en las escaleras, en el banco, en el muro, entre destellos de luz. El color de éstos es distinto según el elemento sobre el que se aplica: amarillo en la piedra, rojo en el banco, blanco en el muro. El material acepta ser transformado por la luz.

Si permanecemos inmóviles frente a la celosía asi stiremos a la constante redefinición del espacio. Cada instante será distinto del anterior y el espacio se expandirá, o reducirá, según el momento del día, y de nuestro espíritu. La materia callada, activada por la luz, genera el dinamismo espacial. El dinamismo, a pesar de los obstinados esfuerzos de la arquitectura contemporánea, no es opuesto a la estabilidad. Precisamente la construcción derivada de las medidas del material permite que la atmósfera espacial se transforme en el tiempo.

El panal de luz, desde una visión más próxima, advierte la infinita matriz de posibilidades construidas con la luz (Fig. 16). Cada una de las celosías se transforma con el tiempo. La luz va esculpiendo su oquedad. Y a cada golpe de cincel define una nueva geometría. Todo ello desde la humildad de una celosía que expresa, sin máscaras, su sencillez geométrica, material y constructiva. Sin embargo, cada imagen esconde una enseñanza. El nivel del suelo del corredor de acceso a la sacristía coincide con el nivel de la cara superior del pozo de agua del patio. A sí se extiende, como una prolongación, hasta recoger los reflejos de la cruz, y su maceta, en el muro opuesto del patio.

Este sólido espejo de agua se sitúa de tal manera que la celosía se prolonga infinitamente sin establecerse un límite entre lo real y lo reflejado. Si detenemos la mirada descubriremos 


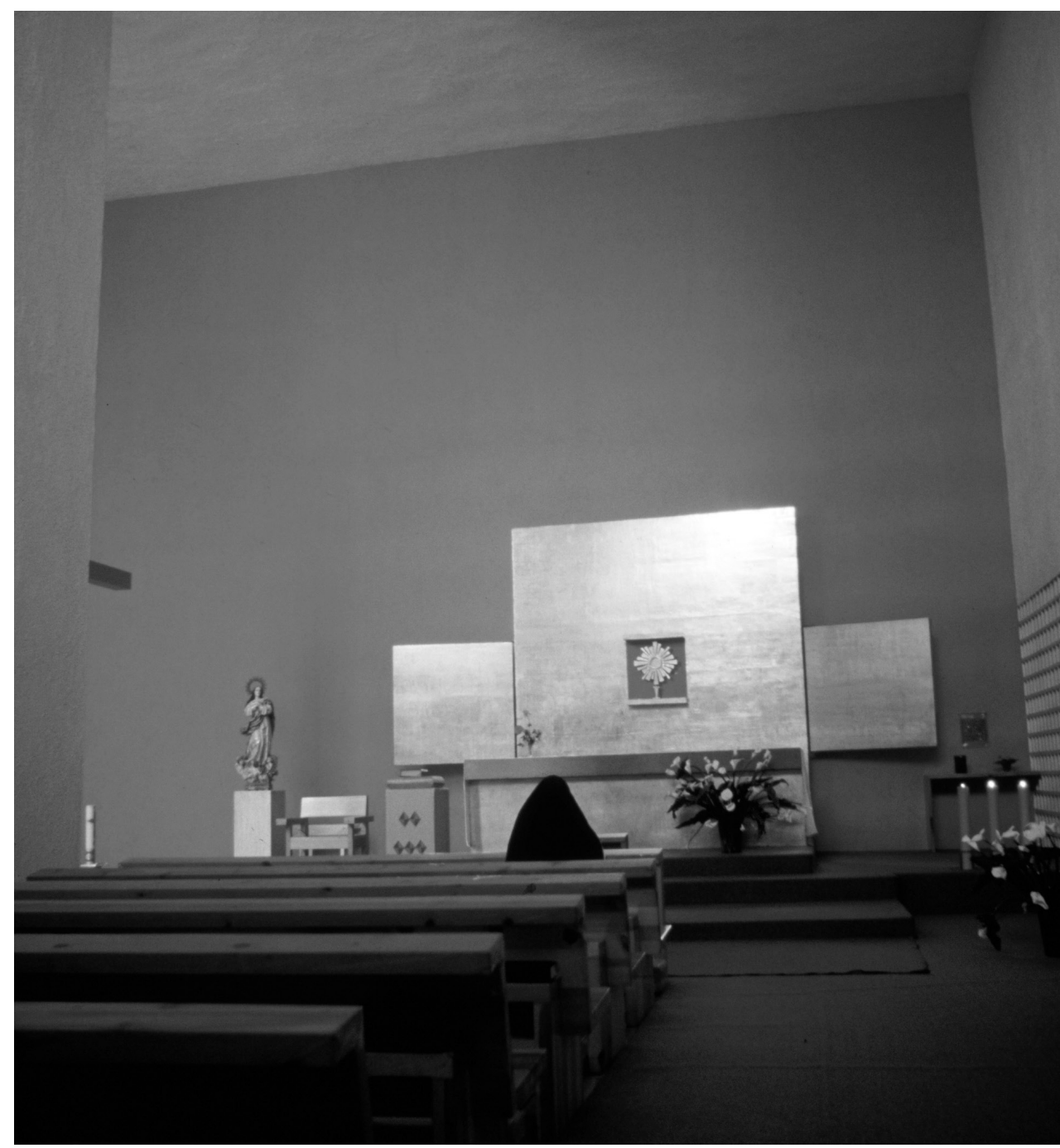

18

que los huecos de la fila inferior de la celosía son equidistantes respecto al espejo de agua y a los huecos de la fila inmediatamente superior (Fig. 17). A sí, la secuencia entre la celosía real, y la reflejada, no introduce ninguna discontinuidad, diluyendo el límite entre ambas realidades. Ésta es la inteligencia al servicio de la condición ilimitada de la arquitectura.

El tránsito a, y desde, la inseguridad es otra de las lecciones de la obra de Barragán. La actitud creativa conlleva la duda. Lo explica Plazaola:

\begin{abstract}
"L a contemplación supone estar atento al ser de las cosas, ver cómo nos habla cada luz, cada color, cada relación de colores, en función del carácter del espacio que proyectamos y del ambiente cultural de nuestro momento y nuestro sitio... Es fácil entender, entonces, que "en el trabajo del artista existe la duda, las incertidumbres, el temor... en todas las etapas de su itinerario no tiene más regla que el tanteo y la prueba. La forma hay que conquistarla; sólo se define en la ejecución. Sólo haciendo puede hallarse y sólo probando puede realizarse; hasta que el acierto final, el único posible, se revele, fulgurante, por sí mismo"16.
\end{abstract}

Por ello Barragán empleaba materiales dúctiles. En colaboración con los artistas Jesús Reyes Ferreira y M athias Goeritz apuesta por una concepción escenográfica de la arquitectura primando la experimentación personal como proceso de creación. De ahí su método de apenas dibujar planos y trabajar en obra, pensando con las manos, como si se tratase de la conformación de un escenario. Esta concepción escenográfica de la arquitectura explica por qué B arragán prefería la piedra, la arcilla y la madera a la alta tecnología del hierro y el hormigón armado. L os material es modernos eran demasiado precisos y determinantes haciendo muy dificultosa la manipulación de los elementos del espacio debido a la inflexibilidad de su ensamblaje racional que tiende a la perfección.
Fig. 18. BARRAGÁN, Luis. Capilla, Convento de las Capuchinas, Tlalpan, Méjico, 1952-1955. (Fotografía C. Labarta).
16. BARRAGÁN, Luis. Discurso de aceptación. Premio Pritzker, Dumbarton Oaks, Washington D.C., 3 de junio de 1980. 
Este valor de la experimentación se relaciona con el hecho de que Barragán apenas hablaba en público sobre arquitectura porque le era imposible teorizar. No contó con el respaldo de una orquestación crítica que explicase y divulgase sus proyectos. No pertenecía a una cultura dominante ni partici paba activamente en los círcul os de poder literarios 0 académicos (la única conferencia que impartió tuvo lugar en Coronado, California, para explicar su trabajo en EI Pedregal). Se recogía, sin más, en la soledad de su estudio de Tacubaya. En realidad, en la deseada soledad de su propia vida.

A sí aunque su arquitectura, como por ejemplo la capilla de Tlalpan, pueda parecer el resultado de una teoría estética minimalista, es, de hecho, el resultado escenográfico de un proceso de prueba y error determinado por la autoridad directora del gusto personal de B arragán (Fig. 18). Porque construye desde la percepción, asociada a la materia, y no exclusivamente desde la geometría. Lo que, necesariamente, implicaba un método de trabajo que, como los artesanos, pudiera confiar en la acción del tiempo sobre la obra como mecanismo de verificación. De ese modo se hacía acompañar de sus amigos artistas e iban construyendo bajo la acción de la percepción superando los límites físicos de los materiales.

Y este construir desde la percepción implica la educación en el arte de ver. Recordemos las pal abras que Barragán dedica al pintor J esús R eyes al recibir el Premio Pritzker: "El arte de ver. Es esencial para el arquitecto y para el paisajista el ver: quiero decir ver más allá del nivel de puro pensamiento racional. Y quisiera rendir homenaje a un gran amigo. M ediante su infalible gusto estético fue un gran maestro en el difícil arte de ver con inocencia"17.

U na inocencia que a B arragán le conduce a las primeras experiencias materiales de la infancia. A caso también por ello comprendió que era preciso olvidar la osadía material, propia de la permanente insatisfacción. Para tornar a la inseguridad de los materiales que permiten la imperfección. Y, con ella, la humana satisfacción de sentirnos acompañados.

Carlos Labarta Aizpún. Arquitecto, ETSA, Universidad de Navarra, 1987. Becado Fulbright Ministerio de Educación y Master in Design Studies, GSD, Universidad de Harvard, 1990; Premio Extraordinario de Doctorado, 2000, en la ETSA de la Universidad de Navarra, escuela en la que realiza su labor docente e investigadora desde la finalización de sus estudios hasta el año 2004 y de la que es Profesor Visitante. Profesor Titular de Proyectos Arquitectónicos en la Escuela de Valladolid (2004-2009) y de la Escuela de Ingeniería y Arquitectura de la Universidad de Zaragoza desde 2009 hasta la actualidad. En 2006 logra la Beca Eisenhower. Su labor investigadora, fundamentalmente sobre la arquitectura del siglo XX, en especial la modernidad arquitectónica, ha sido difundida en revistas especializadas, libros conjuntos así como en diversos congresos internacionales. Obtiene el Premio de Urbanismo del Ayuntamiento de Madrid, 1991. La obra del estudio, publicada en diversos medios, ha sido premiada en sucesivas ediciones del Premio García Mercadal del Colegio de Arquitectos de Aragón, desde 1993 hasta la actualidad, así como en el Premio Ricardo Magdalena del Instituto Fernando el Católico, C.S.I.C., y en la Bienal de Arquitectura Española 2013. Ha obtenido diversos premios en concursos de proyectos como el Primer Premio del Centro de Salud del Actur en Zaragoza, 2000, el Segundo Premio en la Facultad de Ciencias de la Educación de la Universidad de Zaragoza, 2006, o el Tercer Premio del Pabellón Puente, Expo Zaragoza, 2008. Ha participado con su obra en exposiciones conjuntas como "Habitar el Presente", Galería de los Nuevos Ministerios, Madrid, 2007. Es coautor del libro Arquitectura racionalista en Huesca, 2009, y editor de Metodología docente del proyecto arquitectónico, 2011 y de Proyecto arquitectónico y docencia: una cuestión de orden, 2013. 
The Spanish architecture of the eighties and of the first years of the nineties, as seen from Italy, is convincing, but it is not particularly seducing, or at least not until the works of Enric Miralles and Carmen Pinós were published. A new generation of architects attracted by the theories of Koolhaas, by the iconic impact of Nouvel, by the refinement of Herzog and de Meurion and by the hyper-modernism of Hadid, entrusted themselves to the disinhibition of Miralles and Pinós. Let us consider a 1994 project by Aldo Aymonino and a coeval project by ABDR: in both cases, one can sense the attraction for that patchwork of technologies and materials that Miralles and Pinós brought to a paroxysm reminiscent of some of the extreme works of the Catalan Modernism. Their influence is even more evident in one of the most convincing Italian project of these years, namely the Church that Carmen Andriani designed for Rome in 1994, whose layout appears to be the theatre of a conflict between open and close forms, aulic architecture and the vernacular one, attention to large scales and attention to particulars, all of which are conflicts that seem to nourish the architecture of the Catalan architect. Italians also see in Miralles the traces of a modern tradition that is less stiff, more empirical and narrative, more suitable to the new times, namely that of Coderch, Moretti, Leonardo Ricci, Alejandro de la Sota, Gardella and of Fisac, a tradition founded on a non-orthodox modern that continues to tie Italy and Spain deeply. That is not all. Italians also see in Mirales a new urban model. While according to Carlo Aymonino, Aldo Rossi, Vittorio Gregotti and others the urban model is the model of the historical city, whose laws have to be studied both scientifically through typological analysis and artistically through analogy, in the works of Miralles the peripheral city is the new model, marked by fragments of constructions, by large infrastructures and by orographical signs. In essence, for a very short while, the Italian architects identified in Miralles a Mediterranean route to deconstructivism, capable to find, thanks to its iconic force, new expressions to old issues. Some other Spanish architects did not influence the ongoing debate in Italy directly, yet their works present some manifest analogies with some Italian projects. Let us consider the case of Alberto Campo Baeza's well-known Casa Guerrero (2003/2005). It is characterized by an absolutely minimal architecture, designed on a suprematist scheme with some analogies with the hyper-minimal architecture of groups such as Dogma or Baukuh that in the last few years -and in open opposition to the deconstructivist dissipation of forms and signs- has shown an extreme expressive rigor. Yet, that analogy cannot be found in the Caja de Granada Savings Bank (2000/2001), another very well-known work by Baeza, in which the architect designed an architecture characterized by a considerable visual impact without renouncing -as many Italian architects tend to do- the internal space which, on the contrary, is exalted as if it were forged by a primordial act of foundation, thus evoking a bare, ancestral tectonic, similar to the one used by Antón Garcia-Abril -another Spanish architect very appreciated in Italy- who turned bare tectonics into an unmistakable style. Yet, although these architects have a large following in Italy, they do not seem to be exerting a significant influence over the Italian architecture, as it is still scarcely tectonic and, alas, scarcely inclined to create captivating internal spaces.

The influence of Spanish architecture over the Italian one has waned during the last decade, but many Spanish designers are still very appreciated in Italy: José María Sánchez García, Antón Garcia-Abril, Studio Selgas-Cano and RCR are well-known and regularly published, but presently the Italian architecture seems to have taken, with some degree of approximation, some different paths informed by two main currents: architects such as Cino Zucchi, Labics, 5+1aa, Botticini Associati, Archea, C+S and others, who favor an elegantly sober modernism, clear and compact, very composed, although devoid of particular accelerations, belong to the first one. The second current is more radical and keeps together the absolutist architecture that we saw in Dogma and Baukuh and the work of architects such as Paolo Zermani and Renato Rizzi. Between these two main currents there are multiple various experiences that, once again, are proof of the innate eclecticism of our architecture, the eclecticism that we keep on sharing with Spain.

\section{MATTER AND MEMORY. TRANSFORMATIONS IN LUIS BARRAGÁN'S OEUVRE}

Carlos Labarta Aizpún

After a few early years working in the Guadalajara of his birth, Barragán seized a dream opportunity and relocated to Spain's capital city in 1935. In Madrid he embarked on a productive and successful career, designing multi-dwelling units for private developers for 5 years. During that time, further to what he had learnt in his travels across Europe, Barragán saw in modern architecture a catalogue of precise postulates with a reproducible vocabulary. All the buildings he authored in that period indisputably drew from modernist precepts. Nonetheless, like other modern architects, including Le Corbusier himself, Barragán experienced the desolation of white abstraction, of inert and repetitive matter: precise and effective perhaps, but unable to engage with the spiritual values nesting in the deep recesses of the architect's memory.

Around 1940 Barragán decided to break with architectural practice. In so doing he redefined it beyond the stylistic limits that had been his reference until then. The source for that redefinition was the encounter with gardens as the material and object of design, along with the strong attraction exerted on the architect by that specific type of matter. The return to stone, wood or traditional rendering arose not only out of a need to evoke his own experience, but of a desire to conjure up in these materials the memory and nostalgia of other cultures. The El Pedregal gardens and adjacent Prieto House, built in 1945-1950, constituted a veritable laboratory where Barragán, deploying a materially dense architecture, spawned beauty by uniting landscape and aesthetic expression. In a word, the architect aspired to use matter to express humanity's emotional link with nature, its ultimate haven.

With his oeuvre, Barragán blurred the boundaries between technical and humanistic concerns, sensing in every element, every object, the spiritual reasons for building. As in the Capuchin Convent at Tlalpan (1952-1955) and the Gilardi House at Mexico City (19769, with that blurring matter also participates in continuity. Such continuity, converted in his architecture into spatial succession, elicits the feel of a close-knit, changing and uninterrupted sequence. The resulting sleekness is the origin of the joyful, still delight in a work that needs mouldable materials to create that effect. All that together associates memory with the experience of materials. Inherited matter contributes to the generation of timeless and universal architecture, which portrays it not portrayed as the opposite of the spirit but rather, paradoxically, as the vehicle for its expansion.

\section{THE AWAKENING OF MODERN ARCHITECTURE ON MEDITERRANEAN SHORES: DEBATE AND CONTROVERSY IN THE ITALIAN AND SPANISH CONTEXTS}

\section{Pedro Miguel Jiménez Vicario, Micaela Antonucci}

In the period studied (1920-1940), Italian and Spanish journals raised awareness of international architectural movements. Encouraging the dissemination of modern architecture in their respective environs, they became a key to its consolidation. The journals at issue include Architettura e Arti Decorative, founded in 1921 and later renamed Architettura (Organo ufficiale del sindicato degli architetti), headed by Marcello Piacentini; Quadrante, with Pier Maria Bardi and Massimo Bontempelli at the helm (1933-1936); Casabella, managed by Edoardo Persico and Giuseppe Pagano; and Domus, founded in 1928 and run by Gio Ponti until the nineteen forties. In Spain, works authored by May, Taut, Gropius, Schumacher and others were published in any number of journals, such as Arquitectura, Órgano de la Sociedad Central de Arquitectos, Cortijos y Rascacielos, D'Aci i d'Allá, La Gaceta de les Arts, AC and Documentos de actividad contemporánea.

The importance attached by these media to vernacular Mediterranean architecture, in connection in Italy with the debate around Mediterranean culture and in Spain with the national context, sheds light on the impact of the subject on architectural practice, irrespective of the positions adopted by the various journals and the opinions defended by their columnists. As a result of a revisionist approach to the Modernist Movement, a good deal of literature has appeared in recent decades on the development of modern architecture in the Mediterranean context. Drawing from those studies and focusing in particular on documentary sources, the primary aim of this article is to establish the role played by vernacular Mediterranean architecture in the appearance and unfolding of modern architecture in Mediterranean regions through a comparison of developments in Spain and Italy.

\section{FÉRRIZ AND CABRERO: LESSONS FROM AN UNKNOWN AND PARADIGMATIC COLLABORATION BETWEEN A PHOTOGRAPHER AND AN ARCHITEC}

\section{Iñaki Bergera, Cristina Jiménez}

With few exceptions, it is not possible to date collaborations between Asís Cabrero (1912-2005) and a photographer other than Jesús García Férriz (1900-1988). Similarly, there are only few and isolated works proving that Férriz made photo reports of other architects' work. This dual situation points out a unique case study, barely known and unexplored up to date, and of a great theoretical relevance to delve into the intricacies of the marriage architecture \& photography. This situation 